\section{Práticas integrativas e complementares: oferta e produção de atendimentos no SUS e em municípios selecionados}

\author{
Integrative and complementary health practices: the \\ supply and production of care in the Unified \\ National Health System and in selected \\ municipalities in Brazil
}

\author{
${ }^{1}$ Centro de Pesquisas Aggeu \\ Magalhães, Fundação \\ Oswaldo Cruz, Recife, Brasil. \\ 2 Escola Nacional de Saúde \\ Pública Sergio Arouca, \\ Fundação Oswaldo Cruz, Rio \\ de Janeiro, Brasil. \\ ${ }^{3}$ Centro de Ciências da \\ Saúde, Universidade \\ Federal de Santa Catarina, \\ Florianópolis, Brasil. \\ Correspondência \\ I. M. C. Sousa \\ Centro de Pesquisas Aggeu \\ Magalhães, Fundação \\ Oswaldo Cruz. \\ Av. Professor Moraes Rego s/n, \\ Campus da UFPE, Recife, $P E$ \\ 50670-420, Brasil. \\ islandia@cpqam.fiocruz.br
}

\begin{abstract}
The world of Traditional/Complementary and Alternative Medicine has grown and its importance has been emphasized in several studies. In Brazil, the National Policy on Integrative and Complementary Practices encourages their inclusion and empowerment in primary care. This study attempted to identify the provision of services and integrative practices in the Unified National Health System and the production of consultations from 2000 to 2011, contrasting the analysis of available information in national databases with the primary care data collected locally in Campinas (São Paulo State), Florianópolis (Santa Catarina State), and Recife (Pernambuco State). Analysis of the data revealed a mismatch between records in information systems and actual practices in these cities. This mismatch is due largely to lack of definition on the scope of what are understood as integrative and complementary practices in the Brazilian National Policy, thereby posing a major limitation to their measurement and evaluation, since current information does not allow adequate recording of such practices.
\end{abstract}

Traditional Medicine; Complementary Therapies; Health Services; Information Systems; Primary Health Care
Islândia Maria Carvalho de Sousa 1,2 Regina Cele de Andrade Bodstein 2 Charles Dalcanale Tesser 3

Francisco de Assis da Silva Santos 1 Virginia Alonso Hortale 2

\section{Introdução}

A Organização Mundial da Saúde (OMS) estimula, desde o final da década de 70, a implantação da chamada Medicina Tradicional ou Medicina Complementar e Alternativa nos Sistemas de Saúde 1,2. Lançou, em 2002 e 2003, documentos e resoluções com orientações para a referida implantação que incluem quatro pilares fundamentais: estruturação de uma política; garantia de segurança, qualidade e eficácia; ampliação do acesso; e o uso racional 1,2.

A OMS utiliza o termo Medicina Tradicional para se referir às práticas médicas originárias da cultura de cada país, como por exemplo, a medicina tradicional chinesa, a ayurveda hindu, a medicina unani árabe e a medicina indígena. Para a OMS, nos países onde o sistema de saúde realiza ações com base na biomedicina, a Medicina Tradicional é classificada como Medicinas Tradicionais/Complementares e Alternativas ${ }^{1}$. Esse termo significa um conjunto diversificado de ações terapêuticas que difere da biomedicina ocidental 1,3 que incluem práticas manuais e espirituais, com ervas, partes animais e minerais, sem uso de medicamentos quimicamente purificados (acupuntura, reiki, florais, quiropraxia), atividades corporais (tai chi chuan, yoga, lian gong) 1. Entretanto, não há consenso a respeito dessa nomenclatura, haja vista diferentes realidades e práticas no mundo 3,4,5,6,7,8. No México, usa-se 
o termo Medicina Complementar e Integrativa, em Cuba Medicina Natural e Tradicional, nos Estados Unidos e Canadá Medicina Complementar e Alternativa 1,9. No Brasil, utiliza-se práticas integrativas e complementares.

Uma parte relativamente pequena dessas práticas e saberes - acupuntura, técnicas de meditação, plantas medicinais, entre outros - tem sido testada cientificamente, mas com estudos inconclusivos, devido às evidências escassas sobre sua eficácia 1,4,9,10,11,12. Há também estudos que avaliam as reações adversas e, portanto, a segurança no seu uso (principalmente de plantas medicinais e suplementos alimentares) e que concluem pela necessidade de maior aprofundamento no tema 1,11,12. Tal validação científica é específica para alguns problemas ou doenças, conforme as teorias e a nosografia biomédicas, e impõe limites na avaliação desses saberes e práticas 10,12,13. No geral, isola-se técnicas terapêuticas para doenças ou situações específicas, com métodos que satisfazem ou se aproximam de padrões instituídos pela biomedicina 12,13,14,15,16 Isso certamente limita seu uso a doenças específicas, desconsiderando o potencial dessas práticas para promover a saúde entre populações e grupos culturais que utilizam tais saberes há muitas gerações 13,16,17.

Apesar da polêmica e da diversidade de conceitos e nomenclaturas, a literatura sobre o tema atribui algumas características comuns às Medicinas Tradicionais/Complementares e Alternativas, mesmo que não transportáveis para todo o conjunto e nem para todas as situações ou praticantes, haja vista sua heterogeneidade: a procura de cura e equilíbrio por meio de intervenções que reforçam ou induzem uma resposta natural do organismo 1,17,18; um princípio holístico, com enfoque integral dos problemas de saúde e da vida; a busca do equilíbrio entre a mente, o corpo e seu entorno; a ênfase na saúde 4,18 em lugar da doença e o estímulo ao empowerment 4 . Várias delas têm como pressuposto a existência de uma "dinâmica vital", energia, espírito, "sopro", que está além da noção de funcionalidade fisiológica e orgânica 1,3,7,16, e que pode ser estimulada. Em que pese os profissionais da atenção primária à saúde, especialmente os médicos generalistas ou médicos de família e comunidade poderem e necessitarem exercitar uma visão mais integral e holística 19, é compreensível sua dificuldade, nesse exercício, pois decorre tanto das características do saber biomédico quanto dos contextos organizacionais e institucionais em que a prática se desenvolve no Brasil. Essa dificuldade parece ser menor nas Medicinas Complementar e Alternativa, ou pelo menos em outras racionalidades médicas, notadamente nas vitalistas 16,18 .
Como é comum nas situações de cuidado do ser humano em adoecimento (ou mesmo fora dele), nas Medicinas Complementar e Alternativa também há a possibilidade de ocorrer reações adversas, toxicidade e interações entre medicamentos 11,12; embora lhes seja creditada menor agressividade 12 e menor potencial iatrogênico do que na biomedicina 20 . Entretanto, vale registrar a necessidade de ser criterioso na sua abordagem e uso, considerando que tanto os saberes nativos quanto os saberes científicos são pertinentes.

Para além das diferenças terminológicas e conceituais e dos seus possíveis méritos, as Medicinas Tradicional/Complementar e Alternativa se disseminaram no mundo. Estima-se que no continente africano $90 \%$ da população da Etiópia, $70 \%$ de Benin e Ruanda, e $60 \%$ em Uganda utilizem algum tipo de Medicina Tradicional para satisfazer suas necessidades de saúde 1. Na Europa, o percentual de indivíduos que utilizaram alguma vez a Medicina Complementar e Alternativa representa $31 \%$ na Bélgica e $75 \%$ na França. Na Austrália são 48\% 1,5,11. No Reino Unido, a cada ano, cerca de um em cada dez adultos consulta um médico em Medicina Complementar e Alternativa, e $90 \%$ deste procedimento se realiza fora do Sistema Nacional de Saúde 21. Na Índia e na China, que tiveram sua Medicina Tradicional difundida para outros continentes, essas práticas são realizadas nos níveis primários de atenção 20 . No Canadá, estima-se que $70 \%$ da população fazem uso de algum tipo de Medicina Complementar e Alternativa 1,9. Nos Estados Unidos, em 1997, o número de atendimentos pelos chamados "curandeiros" (healers) foi quase o dobro dos atendimentos realizados nos níveis primários de atenção (629 milhões) 4, e em 2007, quatro em cada dez adultos afirmaram ter utilizado algum tipo de Medicina Complementar e Alternativa 1,9. Para os países em desenvolvimento faltam dados, embora existam indícios de que a população e os profissionais dos serviços públicos de saúde utilizem de forma significativa as Medicinas Tradicional/Complementar e Alternativa nos serviços, para atender às necessidades de saúde desses usuários 1,3,18,20.

No Brasil, em 2006, foi estabelecida no Sistema Único de Saúde (SUS) a Política Nacional de Práticas Integrativas e Complementares 3. Uma das suas prioridades foi a inserção e o fortalecimento das práticas integrativas e complementares no nível primário de atenção, com a explicitação dos instrumentos, técnicas e práticas terapêuticas a serem implantados no SUS 3,7. Todavia, ainda existem dificuldades de várias ordens para se conhecer sua implantação. Entre elas se destaca a insuficiência de dados de pro- 
dução e de pesquisas, as limitações no controle destas práticas, a pouca formação e a carência de especialistas 1,3 .

Segundo dados do Ministério da Saúde do Brasil 3, em 2008 existiam no país mais de 800 municípios realizando algumas práticas integrativas e complementares. Ainda que se conheça pouco acerca da Política Nacional de Práticas Integrativas e Complementares e a oferta destas práticas nos serviços de saúde, algumas experiências já ocorriam no país, como por exemplo, nos municípios de Campinas (São Paulo) 3, Florianópolis (Santa Catarina) 22 e Recife (Pernambuco) 23, entre outros 3,18,24. Esse cenário leva a indagar quais as características das práticas integrativas e complementares implantadas nos últimos anos no nível primário de atenção, e o que pode ser visualizado nos sistemas de informação em saúde.

Considerando o contexto referido, este trabalho se propôs a analisar as características da oferta e producão de práticas integrativas e complementares no SUS entre os anos 2000 e 2011, com base em bancos de dados nacionais sobre oferta, profissionais e produção, vis-à-vis dados primários da atenção primária em saúde, coletados nos municípios de Campinas, Florianópolis e Recife.

\section{Metodologia}

Este artigo é resultado do subprojeto da pesquisa Práticas Integrativas e Complementares no Programa de Saúde da Família Voltadas para Atenção à Saúde Mental: Estudos de Caso no Nordeste, Sul e Sudeste. Nesse estudo, de natureza exploratória, os dados referentes aos anos 2000 a 2011 e aos municípios de Florianópolis, Campinas e Recife foram obtidos no Cadastro Nacional dos Estabelecimentos de Saúde (CNES) e no Sistema de Informações Ambulatoriais (SIA), considerando o período em que as consultas em homeopatia e acupuntura passaram a ser incluídas na tabela de procedimentos do SUS.

O CNES é a base cadastral dos sistemas de informação em saúde e registra aspectos da área física, recursos humanos e outros relativos ao funcionamento dos estabelecimentos de saúde nas esferas federal, estadual e municipal. Essa base foi acessada entre os dias 3 e 27 de agosto de 2009, e 10 de maio de 2012 (http://cnes.datasus. gov.br, http://cnes.datasus.gov.br/Mod_Ind_Es pecialidades.asp e http://www2.datasus.gov.br/ DATASUS/index.php?area=0204). Para análise da evolução da oferta entre 2000 e 2011, os dados coletados referem-se ao mês de dezembro de cada ano com as seguintes variáveis: serviços em práticas integrativas e complementares por estabelecimento de saúde e tipo de prestador, Classificação Brasileira de Ocupações (CBO) de profissionais em práticas integrativas e complementares com e sem atendimento no SUS, tipo de vínculo dos profissionais em práticas integrativas e complementares. A lista oficial das práticas na Política Nacional de Práticas Integrativas e Complementares inclui: homeopatia, medicina tradicional chinesa (acupuntura), medicina antroposófica, plantas medicinais e fitoterápicos, termalismo social e crenoterapia 24 . No CNES essas práticas são classificadas como serviço especializado de práticas integrativas e complementares, e pode ser cadastrado mais de um serviço em um estabelecimento de saúde. Os profissionais cadastrados no CNES foram identificados por meio da CBO (versão 2007): acupunturista (322.105); médico acupunturista (223.101); médico homeopata (223.135); psicólogo clínico/psicólogo acupunturista (251.510). Também foram consultadas as CBO de outros profissionais autorizados a oferecer práticas integrativas e complementares: enfermeiro, farmacêutico, cirurgião dentista e fisioterapeuta. Para efeito comparativo, realizou-se o cruzamento entre vínculo e carga horária dos profissionais cadastrados no CNES para os municípios do estudo, em relação ao número de profissionais no país.

O SIA oferece instrumentos para o cadastramento, controle orçamentário, cálculo da produção ambulatorial, além de registrar dados voltados para o gerenciamento da capacidade instalada e produzida, dos recursos repassados aos prestadores de serviços públicos e conveniados ao SUS. Para o período do estudo foram selecionadas para o Brasil e os municípios avaliados as seguintes variáveis, por "local de residência" e "quantidade apresentada" por ano: consultas em homeopatia e acupuntura, práticas corporais em medicina chinesa, sessão de acupuntura com aplicação de ventosas e moxa, sessão de acupuntura com inserção de agulhas, sessão de eletroestimulação.

A seleção dos municípios baseou-se nas experiências distintas das práticas integrativas e complementares na atenção primária em saúde, e por terem iniciado suas atividades nestas práticas antes da edição da Política Nacional de Práticas Integrativas e Complementares. Em Campinas se distribuíam em unidades básicas de saúde; em Florianópolis em algumas equipes de saúde da família e, no caso da acupuntura, em duas policlínicas; em Recife em uma unidade especializada em práticas integrativas e complementares referência para a Estratégia Saúde da Família (ESF) e no Núcleo de Apoio Matricial às Práticas Integrativas. Entre os meses de setem- 
bro de 2009 e janeiro de 2010, foi aplicado um questionário semiestruturado aos gestores municipais, gerentes de unidades de saúde de nível primário de atenção e profissionais das equipes de saúde da família com questões acerca da oferta em práticas integrativas e complementares na atenção primária.

Para análise dos dados foram utilizados os programas Excel 2007 (Microsoft Corp., Estados Unidos) e Statistica 6.0 (Statsoft Inc.; http:/ / www. statsoft.com). Para os dados coletados nos sistemas de informações foi realizada análise de consistência. Parte das respostas ao questionário foi usada de forma complementar à análise. O projeto recebeu aprovação do Comitê de Ética da Fundação Oswaldo Cruz, sob o número 30/2009.

\section{Resultados}

Oferta de serviços de práticas integrativas e complementares no Brasil

O primeiro resultado que chama a atenção foi o de não haver registro de cadastramento de serviços de práticas integrativas e complementares no CNES entre os anos 2000 e 2006. Apesar das consultas de acupuntura e homeopatia serem registradas no SIA desde 1999, observou-se que o cadastramento destes serviços no CNES iniciou após 2006 e que, entre 2007 e 2011, o número de serviços cadastrados no país aumentou de 505 para 3.565, ou seja, 7,06 vezes, com predominância das práticas corporais e acupuntura.

Como um estabelecimento de saúde podia oferecer mais de um tipo de serviço em práticas integrativas e complementares (ambulatorial ou hospitalar), $87 \%$ eram públicos, em sua maioria centros de saúde e unidades básicas de saúde, dos quais, $57 \%$ ofereciam procedimentos de práticas corporais. A Tabela 1 apresenta a distribuição dos serviços no Brasil, por tipo de prestador, para os anos 2007 e 2011.

Oferta de serviços de práticas integrativas e complementares em Campinas, Florianópolis e Recife

De acordo com os dados do CNES, existiam em 2007 serviços cadastrados em práticas integrativas e complementares em Campinas e em Recife. Em Florianópolis, esses serviços passaram a ser cadastrados a partir de 2008, com 59 serviços, e 168 em 2011. Durante esses anos, a oferta de serviços de práticas integrativas e complementares teve crescimento distinto nos municípios, respectivamente, de 2 a 17 em Recife, e de 57 a 101 em Campinas. Em 2011, 286 desses serviços estavam cadastrados em 102 estabelecimentos de saúde dos três municípios. O serviço práticas corporais era em maior número (129), seguido de outras técnicas em Medicina Tradicional Chinesa (77), acupuntura (53) e homeopatia (29); os serviços de medicina antroposófica e termalismo/crenoterapia não estavam cadastrados. No Município de Recife, os serviços de fitoterapia e homeopatia não estavam cadastrados.

Na comparação dos três municípios com o Brasil, observou-se concentração do serviço "práticas corporais" em Florianópolis, presente em mais de $90 \%$ dos estabelecimentos de saúde, dentre os quais, três centros de atenção psicossocial. Em Recife, uma clínica especializada (referência para atenção em práticas integrativas e complementares) e três hospitais. A acupuntura e a homeopatia concentravam-se em maior número nas clínicas privadas. Já as "práticas corporais" concentravam-se nos centros de saúde e unidades de nível primário de atenção.

\section{Profissionais das práticas integrativas} e complementares no Brasil

Entre 2009 e 2010 foram incluídas na CBO as seguintes ocupações: fisioterapeuta acupunturista (223.650), fisioterapeuta quiropraxista (223.645), técnico em quiropraxia (322.125), terapeuta holístico (322.125) e técnico em acupuntura (322.105). Esse último substituiu a CBO acupunturista, impossibilitando o profissional de nível superior de ser cadastrado como acupunturista. Por outro lado, a classificação psicólogo clínico/ psicólogo acupunturista (CBO 251.510) impossibilitou identificar no CNES se este profissional era acupunturista ou clínico e qual a sua atividade no estabelecimento de saúde em que estava cadastrado. O mesmo ocorreu com os enfermeiros, farmacêuticos e cirurgiões-dentista, cujas classificações não contemplavam o detalhamento necessário que evidenciasse a relação com as práticas integrativas e complementares.

Só foi possível comparar a oferta de profissionais no CNES, entre 2007 e 2011, nas seguintes ocupações: acupunturista, médico homeopata, médico acupunturista. Embora o número de profissionais tenha aumentado entre 2007 (2.927) e 2011 (4.368), correspondia principalmente aos que não atendiam no SUS. Nesse período diminuiu o número de acupunturistas no SUS, devido ao início do registro de novas CBO. Assim, para o total de 4.368 profissionais cadastrados em 2011, 77\% estavam distribuídos entre médicos acupunturistas e homeopatas, e $23 \%$ entre fisioterapeutas acupunturistas, técnicos em acupuntura, técnico em quiropraxia, terapeuta holístico e fisioterapeuta quiropraxista. 
Tabela 1

Número e percentual de serviços de saúde * em práticas integrativas e complementares por tipo de prestador. Brasil, 2007/2011.

\begin{tabular}{|c|c|c|c|c|c|c|c|c|}
\hline \multirow[t]{3}{*}{ Prestador de serviços } & \multicolumn{4}{|c|}{ Público } & \multicolumn{4}{|c|}{ Privado } \\
\hline & \multicolumn{2}{|c|}{2007} & \multicolumn{2}{|c|}{2011} & \multicolumn{2}{|c|}{2007} & \multicolumn{2}{|c|}{2011} \\
\hline & $\mathbf{n}$ & $\%$ & $\mathrm{n}$ & $\%$ & $\mathbf{n}$ & $\%$ & n & $\%$ \\
\hline Acupuntura & 93 & 20 & 484 & 15 & 21 & 54 & 255 & 56 \\
\hline Fitoterapia & 28 & 6 & 61 & 2 & $\star \star$ & $\star \star$ & 8 & 2 \\
\hline Outras técnicas da Medicina Tradicional Chinesa & 68 & 15 & 376 & 12 & 6 & 15 & 34 & 7,5 \\
\hline Práticas corporais & 230 & 49 & 2.064 & 66 & 3 & 8 & 98 & 22 \\
\hline Homeopatia & 38 & 8 & 96 & 3 & 7 & 18 & 47 & 10 \\
\hline Termalismo & 3 & 1 & 21 & 1 & 1 & 2,5 & 4 & 1 \\
\hline Medicina Antroposófica & 6 & 1 & 10 & 1 & 1 & 2,5 & 7 & 1,5 \\
\hline Total & 466 & 100 & 3.112 & 100 & 39 & 100 & 453 & 100 \\
\hline
\end{tabular}

Fonte: Cadastro Nacional dos Estabelecimentos de Saúde, Ministério da Saúde, mês de referência dezembro (http://cnes.

datasus.gov.br, http://cnes.datasus.gov.br/Mod_Ind_Especialidades.asp).

* Inclui os prestadores filantrópicos que corresponde a aproximadamente 1\% dos prestadores privados;

** Não houve o registro de serviços no período.

O número de profissionais cadastrados em estabelecimentos que não atendiam ao SUS no Brasil era maior em todas as ocupações, principalmente para os médicos acupunturistas e homeopatas (Tabela 2).

\section{Profissionais das práticas integrativas e complementares em Campinas, Florianópolis e Recife}

Em 2011, nos municípios selecionados e nas classificações relacionadas às práticas integrativas e complementares, foram encontrados 211 profissionais, dos quais $83 \%$ eram médicos acupunturistas e homeopatas e $17 \%$ fisioterapeutas acupunturistas e técnicos em acupuntura e quiropraxia. As mudanças que ocorreram no cadastro dos profissionais nesses municípios entre 2007 e 2011 foram devido ao aumento de médicos acupunturistas e à inserção do técnico em quiropraxia na lista de prestadores privados em Florianópolis e na do CBO de fisioterapeuta acupunturista nos três municípios. Não foi encontrado o registro de fisioterapeuta quiropraxista e terapeuta holístico. A análise por $\mathrm{CBO}$ em 2011 identificou 22 profissionais com duas CBO em práticas integrativas e complementares e 172 com mais de uma CBO, desenvolvendo outra especialidade médica. Quanto à distribuição por municípios, 95 estavam cadastrados em Campinas, 59 em Recife e 57 em Florianópolis. A concentração de médicos acupunturistas e homeopatas seguiu a mesma que no Brasil.
Ao analisar o tipo de prestador (público ou privado) e o vínculo empregatício dos profissionais, observou-se que $42 \%$ tinham vínculo como prestador público e $35 \%$ como prestador público e privado, e $23 \%$ exclusivamente como prestador privado. Desses últimos, $57 \%$ tinham vínculo empregatício.

\section{Produção em práticas integrativas e complementares no Brasil e nos municípios selecionados}

A partir de 2000, foi possível acompanhar a produção de consultas em acupuntura e homeopatia; os procedimentos sessão de acupuntura (ventosa, moxa, eletroestimulação) e práticas corporais só passaram a ser registrados no SIA após 2007. Entre os anos 2000 e 2005, no Brasil, as consultas em homeopatia passaram de 15 para 17 por 10 mil habitantes/ano. No mesmo período, as consultas em acupuntura passaram de 10 para 15 por 10 mil habitantes/ano. Quando comparadas a produção em acupuntura e em homeopatia com as demais práticas integrativas e complementares entre 2006 e 2011, constatouse que as sessões de acupuntura e as práticas corporais tiveram maior registro após a edição da Política Nacional de Práticas Integrativas e Complementares e, no caso das práticas corporais, o registro passou de 1 para 8 por 10 mil habitantes/ ano; e a sessão de acupuntura com inserção de agulhas, de 5 para 56 por 10 mil habitantes/ano (Tabela 3). 
Tabela 2

Número de profissionais em práticas integrativas e complementares inscritos no Cadastro Nacional de Estabelecimentos de Saúde (CNES) por tipo de ocupação e atendimento ao Sistema Único de Saúde (SUS). Brasil, 2007/2011.

\begin{tabular}{|c|c|c|c|c|c|c|}
\hline \multirow[t]{2}{*}{ Ocupação } & \multicolumn{3}{|c|}{2007} & \multicolumn{3}{|c|}{2011} \\
\hline & sus & Não SUS & Total & SUS & Não SUS & Total \\
\hline Acupunturista/Terapeuta natural & 118 & 212 & 330 & * & * & * \\
\hline Fisioterapeuta acupunturista & * & * & * & 247 & 302 & 549 \\
\hline Fisioterapeuta quiropraxista & * & * & * & 1 & 12 & 13 \\
\hline Médico acupunturista & 474 & 693 & 1.167 & 551 & 1.144 & 1.695 \\
\hline Médico homeopata & 560 & 870 & 1.430 & 580 & 1.093 & 1.673 \\
\hline Técnico em acupuntura & * & * & * & 88 & 254 & 342 \\
\hline Terapeuta holístico & * & * & * & 11 & 26 & 37 \\
\hline Terapeuta em quiropraxia & * & * & * & 15 & 44 & 59 \\
\hline Total & 1.152 & 1.775 & 2.927 & 1.493 & 2.875 & 4.368 \\
\hline
\end{tabular}

Fonte: elaboração própria com base no CNES, Ministério da Saúde (situação da base de dados em 10/Mai/2012).

* Não houve o registro de profissionais no período.

Tabela 3

Número de consultas e procedimentos (por 10 mil habitantes) * em práticas integrativas e complementares. Brasil, $2006-2011$.

\begin{tabular}{lcccccc}
\hline Procedimentos & $\mathbf{2 0 0 6}$ & $\mathbf{2 0 0 7}$ & $\mathbf{2 0 0 8}$ & $\mathbf{2 0 0 9}$ & $\mathbf{2 0 1 0}$ & $\mathbf{2 0 1 1}$ \\
\hline Consulta (homeopatia) & 17 & 17 & 16 & 17 & 16 & 15 \\
Consulta (acupuntura) & 17 & 21 & 21 & 19 & 19 & 18 \\
Sessão de acupuntura agulha & $\star \star$ & 5 & 11 & 35 & 19 & 56 \\
Sessão de eletroestimulação & $\star \star$ & 1 & 1 & 2 & 4 & 5 \\
Sessão de acupuntura ventosa & $* \star$ & 1 & 2 & 1 & 2 & 4 \\
Práticas corporais da Medicina Tradicional Chinesa & $\star \star$ & 1 & 6 & 13 & 8 & 8 \\
Total & $\mathbf{3 4}$ & $\mathbf{4 6}$ & $\mathbf{5 7}$ & $\mathbf{8 7}$ & $\mathbf{6 8}$ & $\mathbf{1 0 6}$ \\
\hline
\end{tabular}

Fonte: elaboração própria com base no Sistema de Informações Ambulatoriais, Ministério da Saúde.

* Estimativa da população brasileira realizada pelo Instituto Brasileiro de Geografia e Estatística;

** Não houve registro de produção no período.

Nos três municípios selecionados, entre 2000 e 2005 a produção das práticas integrativas e complementares variou para mais ou para menos quando comparada ao Brasil. Em Campinas, aumentou o número de consultas em acupuntura e homeopatia. Nesse mesmo período, em Florianópolis, as consultas em homeopatia diminuíram de 63 para 41/10 mil habitantes/ano e em Recife, de 28 para 11/10 mil habitantes/ano.

Entre 2006 e 2011, há variação para mais ou para menos em todos os procedimentos. A consulta em homeopatia nos três municípios é a que apresenta maior flutuação, ou seja, em Campinas diminui 34\%, em Recife 60\% e em Florianópolis $82 \%$ (Tabela 4).

\section{Características das práticas integrativas e complementares nos municípios}

Antes de 2006, os três municípios selecionados implantaram as práticas integrativas e complementares, no entanto adaptadas ao contexto local. Em Campinas, desde 2003 elas se distribuem nos serviços de atenção primária. Em Recife, desde 2004 existe uma unidade de saúde denominada Cuidados Integrais em Saúde que funciona como referência para o nível primário de atenção, no qual, a partir de março de 2010, passou a funcionar um núcleo de apoio matricial às práticas integrativas e complementares. Em Florianópolis, as práticas são reali- 
Número de consultas e procedimentos (por 10 mil habitantes) * em práticas integrativas e complementares realizadas em Campinas (São Paulo), Florianópolis (Santa Catarina) e Recife (Pernambuco), Brasil, 2006-2011.

\begin{tabular}{|c|c|c|c|c|c|c|}
\hline Município/Procedimentos & 2006 & 2007 & 2008 & 2009 & 2010 & 2011 \\
\hline \multicolumn{7}{|l|}{ Campinas } \\
\hline Consulta (homeopatia) & 86 & 82 & 58 & 55 & 52 & 55 \\
\hline Consulta (acupuntura) & 66 & 65 & 5 & 3 & 1 & 2 \\
\hline Sessão de acupuntura (agulha) & $\star \star$ & 6 & 7 & 8 & 1 & 7 \\
\hline Sessão de eletroestimulação & $\star \star$ & $\star \star$ & $\star \star$ & $\star \star$ & $\star \star$ & $\star \star$ \\
\hline Sessão de acupuntura ventosa & $\star \star$ & ** & $\star \star$ & 1 & $\star \star *$ & $\star \star$ \\
\hline Práticas corporais da Medicina Tradicional Chinesa & ** & ** & ** & 1 & 2 & 4 \\
\hline Total & 152 & 153 & 70 & 68 & 56 & 68 \\
\hline \multicolumn{7}{|l|}{ Florianópolis } \\
\hline Consulta (homeopatia) & 37 & 13 & 2 & 3 & 7 & 7 \\
\hline Consulta (acupuntura) & 177 & 123 & 3 & 73 & 106 & 109 \\
\hline Sessão de acupuntura (agulha) & $\star \star$ & $\star \star$ & 3 & 72 & 104 & 108 \\
\hline Sessão de eletroestimulação & $\star \star$ & $\star \star$ & ** & $\star \star$ & $\star \star$ & $\star \star$ \\
\hline Sessão de acupuntura ventosa & $\star \star$ & $\star \star$ & ** & 1 & 2 & $\star \star$ \\
\hline Práticas corporais da Medicina Tradicional Chinesa & ** & ** & ** & $\star \star$ & ** & $\star \star$ \\
\hline Total & 214 & 136 & 8 & 149 & 219 & 224 \\
\hline \multicolumn{7}{|l|}{ Recife } \\
\hline Consulta (homeopatia) & 42 & 36 & 36 & 15 & 19 & 17 \\
\hline Consulta (acupuntura) & 19 & 32 & 23 & 47 & 19 & 24 \\
\hline Sessão de acupuntura (agulha) & $\star \star$ & 13 & 25 & 47 & 19 & 29 \\
\hline Sessão de eletroestimulação & ** & 2 & ** & 2 & ** & 3 \\
\hline Sessão de acupuntura ventosa & ** & ** & $\star \star$ & 1 & ** & 1 \\
\hline Práticas corporais da Medicina Tradicional Chinesa & ** & ** & ** & ** & ** & 7 \\
\hline Total & 61 & 83 & 84 & 112 & 57 & 81 \\
\hline
\end{tabular}

Fonte: elaboração própria com base no Sistema de Informações Ambulatoriais, Ministério da Saúde.

* Estimativa da população brasileira realizada pelo Instituto Brasileiro de Geografia Estatística;

** Não houve registro de produção no período.

zadas majoritariamente por profissionais das equipes de saúde da família com especialidade em práticas integrativas e complementares.

Mesmo após a implantação da Política Nacional de Práticas Integrativas e Complementares, constatou-se que dois municípios continuam sem definições normativas sobre suas atividades e ainda com dificuldades para o cadastramento no CNES; inclusive os serviços previstos na normativa nacional e encontrados nos achados empíricos (medicina antroposófica e homeopatia). Os dados mostram profissionais trabalhando como terapeutas holísticos sem o devido registro, apesar de ser possível a criação de uma nova CBO.

É também diferenciado o entendimento do que considera a prática integrativa. Em Campinas, todos os gestores das unidades do nível primário de atenção responderam que ofereciam al- gumas práticas integrativas e complementares, e vários deles citaram como exemplo o artesanato, os grupos terapêuticos e de prevenção (diabetes, hipertensos, obesos, idosos, entre outros). Em Florianópolis, nem todas as práticas corporais são consideradas práticas integrativas e complementares, apesar de terem sido registradas como tal. Atividades como grupos de caminhadas ou de ginástica para idosos ou obesos estão relacionadas a fatores de risco de natureza biomédica e não deveriam ser consideradas práticas integrativas e complementares. O setor de cadastramento dos serviços municipais no CNES justificou a decisão de cadastrar todas as unidades do nível primário de atenção como serviços com práticas corporais, independentemente de serem, para ter um cadastro pronto e, assim, seu registro pudesse se viabilizar de imediato. Dois aspectos a esse respeito são comuns aos demais municípios. Em 
primeiro lugar, foi difícil distinguir práticas corporais terapêuticas, de promoção da saúde e preventivas em geral, ligadas ao cuidado biomédico, das práticas integrativas e complementares. Em segundo lugar, existiam mais serviços de práticas integrativas e complementares nos municípios do que o registrado no CNES. De acordo com as respostas ao questionário, alguns profissionais faziam um "acordo informal" com a coordenação da unidade de saúde para realizar práticas integrativas e complementares, como por exemplo, a dança circular, a shantala e o toque terapêutico em Campinas; o reiki e a acupuntura em Recife; e em várias ESF de Florianópolis.

\section{Discussão}

A Política Nacional de Práticas Integrativas e Complementares favoreceu a visibilidade nos sistemas de informação em saúde das práticas que vinham sendo desenvolvidas, porém sem detalhar o que pode ser registrado como práticas integrativas e complementares. A existência de duas Portarias (nº. 971 de 2006 e n ${ }^{\circ} .853$ de 2006) ${ }^{3}$ pouco esclarecedoras quanto ao termo práticas corporais e outras técnicas, pode caracterizar essa indefinição, favorecendo diferentes maneiras de registro. Essa discussão remete à necessidade de diferenciar sistemas médicos complexos (Racionalidades Médicas) 16,17 de técnicas terapêuticas que atuam de forma complementar à racionalidade biomédica.

O Brasil difere de outros países na oferta de práticas integrativas e complementares, pois predominam as práticas corporais, principalmente nos serviços públicos, o que não coincide com os estudos realizados em países da América Latina $3,20,25$. No SUS, as atividades físicas fazem parte das estratégias para a promoção da saúde e são justificadas pelos estudos internacionais 26 que mostram sua efetividade para alguns fatores de risco à saúde. No entanto, há poucos estudos sobre essas "práticas" de promoção da saúde realizadas nos níveis primários de atenção, incluída a ESF.

É inegável e benéfica a aproximação das práticas integrativas e complementares à política de promoção da saúde 17 . No entanto, a permanência da imprecisão conceitual dificulta o seu registro, mostra a fragilidade na institucionalização dessas práticas e, em consequência, sua avaliação. Em contraste, quando se trata dessa oferta por outros países (China, Índia, Indonésia, Madagascar, Mongólia), os investimentos concentram-se nos níveis primários de atenção e incluem majoritariamente o uso de plantas medicinais, acupuntura e homeopatia 1,20.
Os resultados aqui apresentados mostram que a acupuntura e a homeopatia predominam como oferta do prestador privado e que os procedimentos em acupuntura se expandiram no SUS somente após a permissão do seu exercício por enfermeiros, fisioterapeutas e outros profissionais da saúde 27 , mas a homeopatia continuou com baixa oferta.

Existem poucos serviços de fitoterapia cadastrados no CNES. A política de medicamentos fitoterápicos no SUS é ainda polêmica e tem sido alvo de críticas, principalmente porque há poucos estudos de efetividade, mesmo com os vários incentivos da OMS 1,2 e do Ministério da Saúde do Brasil 3 . Esse aspecto pode mostrar, da mesma forma que em outros países da América Latina 25, que a institucionalização é insuficiente para o seu desenvolvimento.

Avaliar a oferta das práticas integrativas e complementares no SUS pode contribuir com as estratégias que a OMS tem desenvolvido para a expansão das Medicinas Tradicional/Complementar e Alternativa nos países membros 1,2,20. É importante destacar que a maioria dos estudos que discutem as Medicinas Tradicional/Complementar e Alternativa revela pouco acerca da sua oferta nos sistemas de saúde, discute mais seu uso pela população 9,11,21,28,29.

O número de profissionais responsáveis pelas práticas integrativas e complementares cadastrados no CNES é muito menor que o da oferta desses serviços, com exceção da homeopatia, o que pode sugerir que há mais profissionais realizando essas práticas, mas sem registro no sistema. Embora a Portaria no .853 de 200624 tenha tornado possível o registro da produção em práticas integrativas e complementares de outros profissionais, como enfermeiro e cirurgião-dentista, que passaram a registrar o atendimento em acupuntura, a ausência de CBO específica limita esse registro no CNES e consequentemente sua análise, apesar de ter dado visibilidade a categorias profissionais como a fisioterapia 27. É possível que a oferta de profissionais seja maior, considerando que alguns estabelecimentos privados podem não ter se cadastrado. Estudo realizado no Distrito Federal corrobora o presente trabalho ao mostrar que existe uma grande diversidade nas atividades e de profissionais que realizam práticas integrativas e complementares 30 .

Do mesmo modo, o número de médicos acupunturistas e homeopatas cadastrados no CNES se aproxima do relatado na Demografia Médica no Brasil 31. A formação em práticas integrativas e complementares no Brasil é difusa; os cursos são oferecidos por instituições de natureza majoritariamente privada 30 , além da oferta de disciplinas eletivas em algumas universidades federais. Nos 
municípios selecionados, observou-se que as universidades federais oferecem disciplinas eletivas em homeopatia e acupuntura para os cursos de medicina, além de uma residência e curso de especialização. No entanto, a maior oferta de especialização em acupuntura é realizada por instituições de natureza privada.

Nos casos de Florianópolis e Recife, a análise do número de atendimentos das práticas integrativas e complementares no nível primário de atenção foi dificultada porque incluía a rede básica e o nível secundário; diferentemente de Campinas, cujas práticas integrativas e complementares são cadastradas nas unidades básicas de saúde.

Na comparação dos dados do CNES e do SIA para os municípios selecionados, vis-à-vis as respostas aos questionários, foi possível compreender que a oferta é mais diversificada no nível primário de atenção, incluindo, por exemplo, biodança, terapia floral e atividades da dinâmica energética do psiquismo, porém sem códigos disponíveis nos seus formulários. Pode-se supor que a oferta dos serviços esteve atrelada ao apoio da gestão local e interesse dos profissionais especializados, mesmo na ausência de uma política nacional.

Ao comparar o número de profissionais com o cadastro dos serviços em práticas integrativas e complementares nos estabelecimentos públicos, constatou-se que todos os serviços nestas práticas, exceto as corporais, tinham um número significativamente menor de profissionais cadastrados atendendo no SUS. É o caso da homeopatia, com 580 profissionais credenciados em 2011 que informaram vínculo com o SUS, quando só estavam cadastrados 96 serviços públicos em homeopatia no mesmo ano.

A inconsistência dos dados cadastrados no CNES (número de profissionais e número de serviços) evidencia a necessidade de aprimoramento deste banco de dados. A relação entre o número de médicos homeopatas e os serviços públicos em homeopatia merece maior detalhamento, pois a indicação de existir cinco médicos por serviço de homeopatia oferece um retrato diferente do de outros estudos no país 8,22,24,30.

Outro indicador importante, o vínculo profissional, encontrado no nosso trabalho, tem sido pauta de discussões entre o Ministério da Saúde, órgãos colegiados dos secretários municipais e estaduais e órgãos de fiscalização após a publicação da Portaria $n^{o} .134$ de 2011, que limita o número de vínculos a ser registrado no CNES 32 .

Uma das dificuldades para expansão da oferta de práticas integrativas e complementares no SUS diz respeito ao pouco investimento em formação profissional nas instituições de ensino e o explícito interesse das categorias profissionais em tornar cada prática integrativa e complementar uma especialidade 23 . As práticas integrativas e complementares (exceção feita à homeopatia) têm sido um campo de trabalho interdisciplinar, na acepção de Campos \& Domitti 33 de "núcleo e campo de práticas profissionais em saúde". Limitar essas práticas em núcleos por profissões já existentes restringe o acesso e o desenvolvimento das mesmas no SUS e na atenção primária em saúde (embora a capacitação para a prática institucional das práticas integrativas e complementares seja um problema complexo, muito além do escopo deste trabalho).

\section{Considerações finais}

Este estudo, apesar de analisar três municípios em contextos específicos, possibilitou caracterizar o perfil de oferta e produção das práticas integrativas e complementares, revelando, porém, inconsistências nos sistemas de informação e limites nas normatizações vigentes.

O incentivo da Política Nacional de Práticas Integrativas e Complementares para inserção e fortalecimento dessas práticas nos níveis primários de atenção esbarra em alguns obstáculos. O que pode parecer flexibilidade, ou seja, a não definição clara do escopo do termo práticas complementares e integrativas, significa uma grande limitação para a avaliação e o monitoramento de sua oferta e resultados. Os atuais sistemas de informação não permitem o seu registro de forma adequada. Muitas práticas não constam na Política Nacional de Práticas Integrativas e Complementares e não dispõem de códigos nos formulários do CNES e SIA (por exemplo, terapia floral), e os profissionais que realizam procedimentos de práticas integrativas e complementares nesse nível só conseguem fazer o registro em separado, pois o elenco de oferta nestas práticas é classificado como atividades especializadas.

Ficou clara a subnotificação do CNES e o fato de que o Sistema de Informação não conseguiu captar a dinâmica da oferta do nível local. Foi possível constatar que há um distanciamento entre o registrado e o praticado, e algumas vezes isto se deve aos limites impostos por esses sistemas. Outras vezes, há pouca agilidade do município em atualizar as informações e no entendimento conceitual do que são práticas integrativas e complementares. Outro aspecto importante diz respeito à qualidade do dado: aqueles registrados no SIA e CNES não correspondem à oferta e também não há integração entre os referidos sistemas. 
A ênfase na atenção primária para a oferta de práticas integrativas e complementares poderá ser mensurada, monitorada e avaliada, desde que sejam desenvolvidos indicadores e alternativas de registro, o que ainda não ocorreu.

E finalmente, o estudo permitiu identificar um descompasso entre o que se registra nos sis- temas de informação e o que é praticado nos municípios. Esse descompasso pode ser devido, em grande parte, à indefinição do escopo do que se compreende como práticas complementares e integrativas dentro da Política Nacional; o que significa uma grande limitação para sua mensuração e avaliação.

\section{Resumo}

A Medicina Tradicional/Complementar e Alternativa tem crescido no mundo e sua importância vem sendo ressaltada em diversos estudos. No Brasil, a Política Nacional de Práticas Integrativas e Complementares incentiva sua inserção e fortalecimento na atenção primária. Buscou-se identificar a oferta dos serviços e de práticas integrativas e complementares no SUS e a produção de atendimentos entre os anos 2000 e 2011 analisando as informações disponíveis nos bancos de dados nacionais vis-à-vis os dados da atenção primária nos municípios de Campinas (São Paulo), Florianópolis (Santa Catarina) e Recife (Pernambuco). A análise permitiu identificar um descompasso entre $o$ que se registra nos sistemas de informação e o que se pratica nos municípios. Esse descompasso deve-se, em grande parte, à indefinição do escopo do que se compreende como práticas integrativas e complementares na Política Nacional, o que significa uma grande limitação para sua mensuração e avaliação, já que os sistemas de informação atuais não permitem o registro adequado dessas práticas.

Medicina Tradicional; Terapias Complementares; Serviços de Saúde; Sistemas de Informações; Atenção Primária

\section{Colaboradores}

I. M. C. Sousa contribuiu na concepção da pesquisa elaboração e revisão inicial e final do artigo. R. C. A. Bodstein, V. A. Hortale e C. D. Tesser colaboraram na revisão inicial e final do artigo. F. A. S. Santos participou da coleta e análise dos dados nos sistemas de informações, dados empíricos e revisão do artigo.

\section{Agradecimentos}

Ao professor Nelson Filice de Barros (Universidade Estadual de Campinas) pela contribuição na coleta de dados primários. Ao professor Domicio Sá (Fundação Oswaldo Cruz, Pernambuco) pela contribuição na coleta de dados no sistema de informação em saúde. 


\section{Referências}

1. Organización Mundial de la Salud. Estrategia de la OMS sobre medicina tradicional 2002-2005. Genebra: Organización Mundial de la Salud; 2002.

2. Consejo Ejecutivo de la Organización Mundial de la Salud. Resolución EB111.R12/2003. Medicina tradicional. http://whqlibdoc.who.int/eb/2003/ EB111_R12_spa.pdf (acessado em 18/Out/2011).

3. Departamento de Atenção Básica, Secretaria de Atenção à Saúde, Ministério da Saúde. Relatório do 1o Seminário Internacional de Práticas Integrativas e Complementares em Saúde. http://dab.saude. gov.br/semi_praticas_integrativas.php (acessado em 20/Jan/2010).

4. Barrett B, Marchand L, Scheder J, Plane MB, Maberry R, Appelbaum D, et al. Themes of holism, empowerment, access, and legitimacy define complementary, alternative and integrative medicine in relation to conventional biomedicine. J Altern Complement Med 2003; 9:937-47.

5. Nogales-Gaete J. Medicina alternativa y complementaria. Rev Chil Neuro-Psiquiatr 2004; 4:243-50.

6. Vickens A. Complementary medicine. BMJ 2000; 321:683-6.

7. Barros NF, Nunes EDN. Complementary and alternative medicine in Brazil: one concept, different meanings. Cad Saúde Pública 2006; 22:2023-8.

8. Ayers SL, Kronenfeld JJ. Using factor analysis to create complementary and alternative medicine domains: an examination of patterns of use. Health (London) 2010; 14:234-52.

9. McFarland B, Biegelow D, Zani B, Newson J, Kaplan M. Complementary and alternative medicine use in Canada and the United States. Am J Public Health 2002; 10:1616-8.

10. Lewis MBA. Risk and efficacy in biomedical media representations of herbal medicine and complementary and alternative medicine (CAM). Evid Based Complement Altern Med 2011; 16:210-7.

11. Flaherty JH, Takahashi R. The use of complementary and alternative medical therapies among older persons around the world. Clin Geriatr Med 2004; 20:179-200.

12. Süsskind $M$, Thürmann PA, Lüke $C$, Jeschke E, Tabali $\mathrm{M}$, Matthes $\mathrm{H}$, et al. Adverse drug reactions in a complementary medicine hospital: a prospective, intensified surveillance study. Evid Based Complement Altern Med 2012; [Epub ahead of print].

13. Coulter I, Willis E. Explaining the growth of complementary and alternative medicine. Health Sociology Review 2007; 16:214-25.

14. Wearing M. Medical dominance and the division of labour in the health professions. In: Grabich C, editor. Health in Australia: sociological concepts and issues. Frenchs Forest: Pearson Longman; 2004. p. 260-89.

15. Coulter I, Willis E. The rise and rise of complementary and alternative medicine: a sociological perspective. Med J Aust 2004; 180:587-9.
16. Luz MT, Camargo Jr. KR. A comparative study of medical rationatilies. Curare Journal of Ethnomedicine 1997; 12:47-58.

17. Tesser CD. Práticas complementares, racionalidades médicas e promoção da saúde: contribuições poucos exploradas. Cad Saúde Pública 2009; 25:1732-42.

18. Queiroz MS. O itinerário rumo às medicinas alternativas: uma análise em representações sociais de profissionais da saúde. Cad Saúde Pública 2000; 16:363-75.

19. Pietronni P. Beyond the boundaries: relationship between general practice and complementary medicine. BMJ 1992; 305:564-6.

20. World Health Organization. Report of the WHO Interregional Workshop on the Use of Traditiona Medicine in Primary Health Care. Ulaanbaatar: World Health Organization; 2009.

21. Thompson T, Gene F. Complementary therapies and the NHS. BMJ 2005; 331:856-7.

22. Thiago SCS, Tesser CD. Percepção de médicos e enfermeiros da Estratégia de Saúde da Família sobre terapias complementares. Rev Saúde Pública 2011; 45:249-57.

23. Santos FAS, Sousa IMC, Gurgel IGD, Bezerra AFB, Barros NF. Política de práticas integrativas em Recife: análise da participação dos atores. Rev Saúde Pública 2011; 45:1154-9.

24. Departamento de Atenção Básica, Secretaria de Atenção à Saúde, Ministério da Saúde. Política Nacional de Práticas Integrativas e Complementares no SUS. Brasília: Ministério da Saúde; 2006.

25. Nigenda G, Mora-Flores G, Aldama-López S, Orozco-Núñez E. La práctica de la medicina tradicional en América Latina y el Caribe: el dilema entre regulación y tolerancia. Salud Pública Méx 2001; 43:41-51.

26. Pate RR, Pratt M, Blair SN, Haskell WL, Macera CA, Bouchard C, et al. Physical activity and public health. JAMA 1995; 273:402-7.

27. Santos FAS, Gouveia GC, Martelli PJL, Vasconcelos EMR. Acupuntura no Sistema Único de Saúde e a inserção de profissionais não médicos. Rev Bras Fisioter 2009; 13:330-4

28. Eisenberg DM, Kessler RC, Foster C, Norlock FE Calkins DR, Delbancoet TL. Unconventional medicine in the United States. Prevalence, costs, and patterns of use. N Engl J Med 1993; 4:246-52.

29. Álvarez C, Luz S. El uso de medicina alterantiva y medicina tradicional en Medellín, 2005. Revista de La Facultad Nacional de Salud Pública 2007; 25:100-9.

30. Parreiras AMP, Pereira Neto MR. Das Medicinas Tradicionais às práticas integrativas de saúde. Caracterização dos recursos humanos nas práticas alternativas de saúde adotadas no Distrito Federal. Brasília: Universidade Nacional de Brasília/ObservaRH, 2010. 
31. Scheffer M. Demografia médica no Brasil. São Paulo: Conselho Regional de Medicina do Estado de São Paulo/Conselho Federal de Medicina, 2011. http://www.cremesp.org.br/pdfs/demografia_2 dezembro.pdf (acessado em 16/Mai/2012).

32. Conselho Nacional dos Secretários de Saúde. Boletim virtual. http://www.conass.org.br/ aplicacoes/boletim/dsp_boletimmais.php?id_ textomateria $=1818 \& i d \_b o l e t i m=1090 \& i d$ tema=1092 (acessado em 20/Mai/2011).
33. Campos GWS, Domitti AC. Apoio matricial e equipe de referência: uma metodologia para gestão do trabalho interdisciplinar em saúde. Cad Saúde Pública 2007 ; 23:399-407.

Recebido em 30/Ago/2011

Versão final reapresentada em 30/Jul/2012 Aprovado em 17/Ago/2012 